Disclosure of Interests: None declared

DOI: 10.1136/annrheumdis-2020-eular.1822

\section{FRI0652-HPR DEVELOPMENT OF THE ADVANCED PRACTICE NURSING CONSULT IN RHEUMATOLOGY IN THE MULTIDISCIPLINARY APPROACH OF INFLAMMATORY DISEASES MEDIATED BY IMMUNITY}

A. Lopez Esteban ${ }^{1,2}$, P. Morales de Los Ríos Luna ${ }^{1}$, P. Villalpando $G^{2}$, A. López Calleja $^{1}$, T. Del Río Blasco ${ }^{1}$, B. Villarrubia Martín², S. Sánchez Vázquez², C. Lobo Rodríguez ${ }^{1}{ }^{1}$ Hospital General Universitario Gregorio Marañón, CEIMI, Madrid, Spain; ${ }^{2}$ Hospital General Universitario Gregorio Marañón, Madrid, Spain

Background: Patients with immunity mediated inflammatory diseases (IMID) often have clinical manifestations and comorbidity in the field of various medical specialties. A center has been created in our hospital for the comprehensive care of patients with IMID. It is an innovative healthcare model, that incorporate patients into its governance. Physicians, pharmacists and advanced practice nurses (APN), collaborates in consultation or in the day hospital (DH).

Objectives: To analyze the activity of the rheumatology APN consult integrated in the multidisciplinary team, and the impact on health care and quality of life on IMID patients.

Methods: Descriptive study of the rheumatology APN activity since the opening of the center for a year. The APN consultations were face-to-face (scheduled or demanded) or by telephone. Variables measured: demography, diagnosis, treatments, clinic activity and patient reported outcomes (PRO). In the face-to-face consultation, was included: integral valuation (clinic, functional and psychosocial), education for Health (information about disease and treatments, adverse effects, healthy living habits), drug administration, emotional support. In the telephone consultation, the rheumatology APN is the reference professional for monitoring, question solving and advice in case of flare or adverse event.

Results: 721 patients were evaluated, mean age 54.6 years, (range, SD) (20$90,13.9), 61.3 \%$ women, with a total of 1737 consults. Diagnosis: 324 (44.9\%) RA, 221 (30.6\%) SpA, 100 (13.9\%) PsA and 76 (10.5\%) other diseases. Treatment modality: IV 293 (40.4) SC 399 (55.3\%) and oral 29 (4.0\%). Rheumatology APN activities are described in Table 1. 1415 face-to-face consultation were made, $82.7 \%$ scheduled and $17.2 \%$ demanded either by the patient, the rheumatologist or another member of the multidisciplinary work-team. Among the face-to-face consultations, 62 (4.4\%) patients were attended the same day in the medic consult due to disease flare or other disease problems, $38(2.7 \%)$ patients were sent to another specialist of the work-team due to co-morbidity. The activity executed in the rheumatology APN consultation is shown in Table 2

Table 1. Educative interventions by the APN.

Information about the disease -Information about the treatment

-Medicine administration

-Adverse effects of the medicine

-Healthy life style habits

-Vaccines

-Evaluation of cardiovascular risk

-Signs and symptoms of alerts due to the pathology

- Ongoing following of the evolution of the disease.

-Support for the achievement of the treatment and possible difficulties.

Table 2. Activity done in the consult. $N^{\circ} 1.737$

\begin{tabular}{|c|c|c|c|}
\hline Face-to-face activity & $\begin{array}{c}\text { № consults } \\
1.415\end{array}$ & Non face-to-face activity & $\begin{array}{c}\text { № consults } \\
322\end{array}$ \\
\hline $\begin{array}{l}\text { Complete valuation due to } \\
\text { necessities }\end{array}$ & $721(51 \%)$ & Valuation & $58(18.0 \%)$ \\
\hline Educative Interventions & $1.305(92.2 \%)$ & Questions & $96(29.8 \%)$ \\
\hline Valuation and following & $1.372(97.0 \%)$ & Test result & $26(8.1 \%)$ \\
\hline PRO execution & $1.156(81.7 \%)$ & & \\
\hline Articular counting's & $733(51.8 \%)$ & & \\
\hline Valuation of the diseases activity & $737(52.1 \%)$ & Process of appointment & $105(32.61$ \\
\hline •DAS28 PCR & $419(26.6 \%)$ & -Requested by the doctor & $50(47.0 \%)$ \\
\hline •ASDAS-PCR & & -Requested by the patient. & $55(53.0 \%)$ \\
\hline Cardiovascular risk valuation. & $721(51.0 \%)$ & $\begin{array}{l}\text { Derivative to different medi- } \\
\text { cal consults }\end{array}$ & $37(11.5 \%)$ \\
\hline Blood extraction & $724(51.2 \%)$ & Solved by APN & $285(88.5 \%)$ \\
\hline Analytics monetarization & $1.171(82.8 \%)$ & & \\
\hline Medicine monetarization & $1.372(97.0 \%)$ & & \\
\hline
\end{tabular}

Day Hospital Management
Conclusion: The rheumatology APN takes a vital part in the multidisciplinary team, offering a holistic approach, as well as efficient and high-quality care, offer ing quick response, reducing waiting time and becoming an important part of this patient-centered model.

Disclosure of Interests: None declared

DOI: 10.1136/annrheumdis-2020-eular.3257

\section{FRIDAY, 05 JUNE 2020}

HPR Interventions (educational, physical, social and psychological)

\section{FRI0653-HPR AN OLEUROPEIN-BASED DIETARY SUPPLEMENT IMPROVES JOINT FUNCTIONALITY IN OLDER PEOPLE WITH HIGH KNEE JOINT PAIN}

M. N. Horcajada ${ }^{1}$, M. Beaumont ${ }^{2}$, N. Sauvageot ${ }^{3}$, L. Poquet ${ }^{1}$, M. Saboudjian ${ }^{1}$, A. C. Hick ${ }^{4}$, B. Costes ${ }^{4}$, L. Garcia ${ }^{4}$, Y. Henrotin ${ }^{5} .{ }^{1}$ Nestle Research, EPFL Innovation Park, Lausanne, Switzerland; ${ }^{2}$ Nestle Research, Clinical Development Unit, Lausanne, Switzerland; ${ }^{3}$ Nestle Research, Clinical Development Unit, Lausanne, Switzerland; ${ }^{4}$ Artialis SA, Liège, Belgium; ${ }^{5}$ University of Liège, Liège, Belgium

Background: OLE provides oleuropein the most prevalent phenolic componen in olive leaves and has been shown to have potent anti-inflammatory and anti-oxidant effects potentially interesting for joint health (1).

Objectives: The aim of this study was to investigate the effects of a 6-month intervention with an Olive Leaf Extract (OLE) standardized for oleuropein content on knee functionality and biomarkers of bone/cartilage metabolism and inflammation

Methods: The study was a randomized, double-blind, placebo-controlled, multi-centric trial of 124 subjects with mild knee pain or mobility issues. Subjects were randomized equally to receive twice a day one capsule of either maltodextrin (control treatment, CT) or 125-mg OLE (BonoliveTM, an Olive Leaf Extract containing $50 \mathrm{mg}$ of Oleuropein) for 6 months. The co-primary endpoints were Knee injury and Osteoarthritis Outcome Score (KOOS) using a self-administered questionnaire and serum Coll2-1NO2 specific biomarker of cartilage degradation. The secondary endpoints were each of the five sub-scales of the KOOS questionnaire, Knee pain VAS score at rest and at walking, OARSI core set of performance-based tests and serum biomarkers (Coll2-1, MPO, CTX1, osteocalcin, PGE2 and Vplex cytokines assay in serum) and concentration of Oleuropein's metabolites in urine.

Results: Primary (global KOOS score, biomarker Coll2-1 NO2) and secondary endpoints (the five subscales of the KOOS score) improved time dependently in both groups. OLE treatment showed significantly elevated urinary oleuropein metabolites (oleuropein aglycone, hydroxytyrosol, homovanillyl alcohol and isomer of homovanillyl alcohol), and was well tolerated without significant differences in number of subjects with adverse events. At 6 months, OLE group showed a higher global KOOS score compared to placebo (treatment difference $=3.73 ; 95 \% \mathrm{Cl}=[-4.08 ; 11.54] ; \mathrm{p}=0.34)$, without significant changes of inflammatory and cartilage remodeling biomarkers. Subgroup analyses demonstrated a large and significant treatment effect of OLE in subjects with high walking pain at baseline $(14.4 ; 95 \% \mathrm{Cl}=[1.19 ; 27.63], \mathrm{p}=0.03)$. This was observed at 6 months for the global KOOS score and each different subscale and for pain at walking $(-23.07 ; 95 \% \mathrm{Cl}=[-41.8 ;-4.2] ; \mathrm{p}=0.02)$. These treatment effects at 6 months were significant for KOOS score as well as for the subscales Pain and QoL and the pain at walking.

Conclusion: OLE was not effective on joint discomfort in people with low to moderate pain at baseline but significantly benefited subjects with high pain at treatment initiation. As oleuropein is well-tolerated, OLE can be used to relieve knee joint pain and enhance mobility in subjects with articular pain the most painful subjects.

References:

[1] Horcajada MN, Sanchez C, Membrez Scalfo F, Drion P, Comblain F, Taralla $S$, Donneau AF, Offord EA, Henrotin Y. Oleuropein or rutin consumption decreases the spontaneous development of osteoarthritis in the Hartley guinea pig. Osteoarthritis Cartilage. 2015 Jan;23(1):94-102

Disclosure of Interests: Marie-Noelle Horcajada Employee of: nestlé, Maurice Beaumont Employee of: nestle, Nicolas Sauvageot Employee of: Nestlé, Laure Poquet Employee of: Nestlé, Madleen Saboudjian Employee of: Nestlé, Anne-Christine Hick Employee of: Artialis SA, Berenice Costes Employee of: Artialis SA, Laetitia Garcia Employee of: Artialis, Yves Henrotin Grant/research support from: HEEL, TILMAN DOI: 10.1136/annrheumdis-2020-eular.3655 